\title{
System und Methode - Zur methodologischen Begründung transzendentalen Philosophierens in Fichtes »Begriffsschrift«
}

\author{
Jürgen Stahl (Leipzig)
}

Ueber den Begriff der Wissenschaftslehre - die Aufhebung der Propädeutik in der philosophisch-methodologischen Begründung

Unmittelbar im Zusammenhang mit der Entfaltung seiner philosophischen Auffassungen in den Züricher Vorlesungen und im Vorfeld der Erarbeitung der Grundlage der gesammten Wissenschaftslehre entstand Anfang des Jahres 1794 Fichtes kleinere Schrift Ueber den Begriff der Wissenschaftslehre oder der sogenannten Philosophie, als Einladungsschrift zu seinen Vorlesungen über diese Wissenschaft. Deren Zweckbestimmung bezeichnete Fichte vier Jahre später anläßlich des Erscheinens der zweiten Auflage als die »einer Einleitung in dieses System «. ${ }^{1}$ Diese Funktion erfüllt der Text nach eigener Aussage des Philosophen insofern, als darin die Möglichkeit einer genetischen Ableitung der Inhalte des Bewußtseins und die Regeln einer solchen Wissenschaft der »Kritik «, der methodologischen Betrachtung, unterzogen wurde.

Eine philosophiehistorische Würdigung der Schrift ist sicher weniger dadurch zu leisten, daß man sie überschwenglich zur »Programmschrift des deutschen Idealismus « schlechthin erhebt. ${ }^{2}$ Denn die Originalität der Fichteschen Leistung erwuchs auf einem spezifischen historischen Hintergrund: Den

1. J.G. Fichte: Ueber den Begriff der Wissenschaftslehre oder der sogenannten Philosophie, als Einladungsschrift zu seinen Vorlesungen über diese Wissenschaft. [1794], GA I/2, 159.

2. V. Hösle: Hegels System. Der Idealismus der Subjektivität und das Problem der Intersubjektivität, Bd. 1, Systementwicklung und Logik, Hamburg 1988, S. 23. 
methodologischen Diskussionen in der deutschen und europäischen Aufklärung ebenso wie dem vielfältigen und zeitgleich sich vollziehenden Diskurs um die von Kant inaugurierte Problemstellung. ${ }^{3}$

Im Konnex der systematischen Entfaltung der transzendentalphilosophischen Position in der Grundlage der Wissenschaftslehre bildet die Arbeit Ueber den Begriff der Wissenschaftslehre die in Fichtes Sicht unumgängliche methodologische Reflexion. Der im November 1793 in der Rezension "Gebhard « erstmals explizierte und in Auseinandersetzung mit den Auffassungen Kants, Maimons, Schulzes und Reinholds originär entwickelte transzendentale Standpunkt wurde eingebettet in einen sich in Wechselwirkung mit der Systemausarbeitung neu formierenden Begriff von Wissenschaft. Das Motiv für diese Reflexionen waren keineswegs lediglich äußerliche Gelegenheitsgründe, sondern sie folgten einer inneren Notwendigkeit zum Erlangen theoretischer Klarheit über die Frage nach der Möglichkeit von Wissenschaft. Fichtes Gedanken sind dabei zum einen geprägt von den gängigen Diskussionen seiner Zeit - resümiert in den drei grundsätzlichen Forderungen an eine wissenschaftliche Theorie: »Eine Wissenschaft hat systematische Form; alle Sätze in ihr hangen in einem einzigen Grundsatz zusammen, und vereinigen sich in ihm zu einem Ganzen [...]. « Zugleich erklärte Fichte diese in der Descarteschen Tradition stehenden und in Deutschland durch das Wirken der Wolffschen Schule allgemein gewordenen Forderungen für keineswegs ausreichend, formulierte er seinen darüber hinausgehenden Anspruch an Wissenschaft sowohl in bezug auf die methodischen Erfordernisse als auch hinsichtlich der der Wissenschaft zukommenden Rolle im Leben der Gesellschaft. ${ }^{5}$

Der sachliche Fichtesche Anspruch der Arbeit ging darauf aus, zur Grundlegung des Systems der Wissenschaftslehre durch deren metatheoretische Reflexion die Methode transzendentalen Philosophierens zu legitimieren. Was aber bei anderen philosophischen Darstellungen jener Zeit in der Regel in Vorworten mit propädeutischem, zur eigentlichen Thematik hinführendem Charakter aufgenommen ist, erscheint bei Fichte nunmehr als eigenständige Problemstellung mit einer gegenüber der philosophischen Theorie neuen Funktion. Auch Kants Fragestellung: »Wie ist Metaphysik als Wissenschaft

3. So machte W.H. Schrader darauf aufmerksam, daß sich Fichtes Schrift eng an Gedanken Reinholds anlehne. Vgl.: W.H. Schrader: »Systemphilosophie als Aufklärung. Zum Philosophiebegriff K.L. Reinholds«, in: studia leibnitiana, Bd. XV, Wiesbaden 1983, S. 80.

4. Ebenda, S. 112.

5. Vgl.: R. Lauth: „Fichtes revolutionierende neue Konzeption von Philosophie als Wissenschaft«, in: ders.: Transzendentale Entwicklungslinien von Descartes bis zu Marx und Dostojewski, Hamburg 1989, S. 384. 
möglich? «, ist in die Vorrede zur Kritik der reinen Vernunft, in die resümierende und zugleich propädeutische Diskussion über die Möglichkeit der Kritik, ihre Bestimmungen, Quellen, ihre Grenzen und ihren Umfang eingebettet; die gleiche Bestimmung erfährt die Kritik in der Erörterung der »Architektonik der reinen Vernunft ${ }^{6}{ }^{6}$

Fichte nahm mit seiner Frage: "Wie ist Gehalt und Form einer Wissenschaft überhaupt, d.h. wie ist die Wissenschaft selbst möglich? « ${ }^{7}$ die Problemstellung Kants auf. Zugleich erfährt diese durch ihn aber eine entscheidende Verschärfung: In der systembegründenden Funktion der Reflexion über die Möglichkeiten und Mittel der Kritik wird der "vorwissenschaftliche« Charakter abgestreift; die Systembegründung wird dadurch selbst zum Moment der Systemkonstitution und -konstruktion. Die methodologische Reflexion erscheint ebenso als deren Voraussetzung und Bedingung wie sie deren Ergebnis ist, die dadurch selbst wissenschaftlichen Charakter erlangt und so zur "Wissenschaft von der Wissenschaft überhaupt « wird. ${ }^{8}$ Dies ist nicht nur eine isolierte, gelegentliche methodologische Forderung Fichtes - die Entstehungsgeschichte und der inhaltliche Zusammenhang der Arbeiten Ueber den Begriff und Grundlage der gesammten Wissenschaftslehre weisen diesen Funktionswechsel aus.

Ausgangspunkt der methodologischen Erörterung Fichtes war die Feststellung, daß Philosophie und Mathematik in ihren begrifflichen Instrumentarien durch wesentliche Unterschiede geprägt sind, daß die Philosophie »nicht, wie die Geometrie und die Mathematik, überhaupt ihre Begriffe in der Anschauung construiren $[\ldots] \ll$. Dagegen sei die »Form der Deduktion [...] die gleiche, wie sie in der Mathematik gilt, nämlich die von der allgemeinen Logik vorgeschriebene «. ${ }^{9}$ Fichte vollzog auf diese Weise eine apodiktische Abkehr von der die europäische Aufklärung seit dem 17. Jahrhundert durchziehenden Diskussion um die Einheit und Spezifik von mathematischer und philosophischer Erkenntnis im Ringen um die Durchsetzung verbindlicher methodischer Prinzipien für die Erkenntnisgewinnung und den Theorienaufbau. ${ }^{10}$

6. I. Kant: Kritik der reinen Vernunft, B 22, A 841.

7. J.G. Fichte: Ueber den Begriff [...], GA I/2, 117.

8. Ebenda.

9. J.G. Fichte: Brief Nr. 175 vom 15. Januar 1794, GA III/2, 40.

10. Als Beispiel für die sich an der Mathematik bzw. Physik orientierende Diskussion vgl. J.H. Lambert: "Anlage zur Architektonic, oder die Theorie des Einfachen und des Ersten in der philosophischen und mathematischen Erkenntniß«, 2. T. §683, in: J.H. Lambert: Philosophische Schriften, hrsg. v. H.-W. Arndt, 4. Bd, Hildesheim 1965. 
Den Inhalt der »Wissenschaft von der Wissenschaft überhaupt « bilden somit weder unverbindliche Ausführungen über diese oder jene verwendbare Methode. Ihr Inhalt ist die besondere Methodik, die mit den ihr eigenen Mitteln begründet, welche Bestimmungen des menschlichen Erkenntnisvermögens von diesen methodologischen Prinzipien notwendig betroffen sind.

Die Begründung der transzendentalen Logik als methodisches Fundament zur Analyse der Vermögen des menschlichen Geistes

Bereits im Ausgang der europäischen bürgerlichen Aufklärung war deutlich geworden, daß weder die Philosophie noch die aufkeimenden Sozialwissenschaften und auch nicht die Naturforschung dort, wo sie auf die Erklärung des Lebens ausging, als bloßer Formalismus nach dem Modell der Mathematik oder der Physik aufbaubar war. Der philosophische Denkeinsatz 1793/1794 ist in methodologischer Hinsicht vor allem von der Ablösung der formalen Logik durch die transzendentale Logik, der Erneuerung und Weiterbildung der Dialektik - verstanden als die sich entwickelnde menschliche Erkenntnis in methodisch bewußt gewordener Gestalt - zum vorrangigen methodischen Fundament philosophischer Theorienbildung markiert. In der philosophischen Methodologie gelang es Fichte, sowohl über diese als formalisiertes Orientierungswissen als auch als deskriptive Verallgemeinerung des methodischen Vorgehens der einzelnen Wissenschaft hinauszugehen und demgegenüber seine methodologischen Überlegungen als Gesamtheit methodischer Konsequenzen des transzendentalphilosophischen Ansatzes zu entwickeln.

Dieser Positionswandel läßt sich in Fichtes Schrift Ueber den Begriff der Wissenschaftslehre sehr genau ablesen. Unter Abweisung der Mathematik als normatives methodisches Vorbild analysierte er im Rückgang auf Kant die Rolle des erkennenden Subjektes am Beispiel des Naturwissenschaftlers im Erkenntnisprozeß und explizierte seinen transzendentalen Standpunkt. Danach läßt sich erweisen, daß der Naturforscher

"selbst erst die Gesetze der Natur, die er durch Beobachtung von ihr zu lernen glaubt, in sie hineingelegt habe, und daß sie sich [...] vor aller Beobachtung vorher aus dem Grundsatze alles menschlichen Wissens ableiten lassen. Es ist wahr, daß kein Naturgesetz und überhaupt kein Gesetz zum Bewußtseyn kommt, wenn nicht ein Gegenstand gegeben wird, auf den es angewandt werden kann; es ist wahr, daß nicht alle Gegenstände nothwendig, und nicht alle in dem gleichen Grade damit übereinkommen müssen; [...] aber eben darum ist es wahr, daß wir sie nicht durch Beobachtung lernen, sondern sie aller Beobachtung zugrunde 
legen, und daß es nicht so wohl Gesetze für die von uns unabhängige Natur, als Gesetze für uns selbst sind, wie wir die Natur zu beobachten haben. «"

Fichte hob die unvermittelte ontologisch bestimmte Identität von Seins- und Denkgesetzen zugunsten der Akzentuierung des aktiven Verhaltens des Subjekts in der erkennenden Tätigkeit auf. Damit war für Fichte das Verhältnis des Subjektes zum Objekt bzw. Gegenstand der Erkenntnis und das Problem der Erkenntnisgewinnung keine - wie in der Aufklärung — weitgehend unproblematische Beziehung, die keiner besonderen Reflexion bedarf. Statt dessen ist der von ihm herausgehobene Gesichtspunkt der, daß das Subjekt ein theoretisches Modell in der Erklärung des Erkenntnisobjektes erarbeitet und dies seine originäre Leistung darstellt. Und folglich muß das erkennende Subjekt in der Erarbeitung des Modells ebenso notwendig nach den Gesetzen der menschlichen Erkenntnis handeln wie sich die Ergebnisse menschlicher Denkleistung nicht der intersubjektiven Überprüfbarkeit entziehen dürfen.

Aufbauend auf der Auseinandersetzung mit Reinhold, Schulze und Maimon um den systematischen Ort der Vorstellung im System der Handlungsarten des menschlichen Geistes und die Begründung des Ersten Grundsatzes in Gestalt des Ich=Ich als Einheitspunkt sowohl der theoretischen Philosophie als auch zwischen ihr und der praktischen Philosophie erfolgte nun in der $\mathrm{Be}$ griffsschrift die damit notwendig verbundene Begründung des besonderen methodischen Vorgehens. Es ist danach weder der Satz des Widerspruchs, noch der des Grundes der dem methodischen Erfordernis eines absolut Ersten Grundsatzes, der das gesamte Wissen begründen soll und somit der gesamten Wissenschaftslehre gemeinsam sein muß, zu genügen vermag. Über beide hinausgehend bedarf es eines Verfahrens, in dem »Eintheilung [...] nur durch Gegensetzung möglich [ist], deren Glieder aber doch einem dritten gleich seyn müssen. « ${ }^{12}$ Damit war das dialektische Widerspruchsverhältnis als eine qualitative Beziehung zum Ausgangspunkt des in strenger Folgerichtigkeit zu vollziehenden Verfahrens der Entfaltung des Ausgangsverhältnisses in der Deduktion genommen, die sich über ein erneutes quantitatives Einschränken durch den bestimmten Gegensatz konkretisiert. Das Setzen und Lösen dialektischer Widersprüche rückte auf diese Weise in das Zentrum philosophischer Methodik, wobei die Gegensätze sich nicht in beliebiger Relation zueinander befinden, sondern das Eine nur in Beziehung auf sein Anderes und das Ganze nur durch und in dieser Beziehung existent ist. 
Ebenso geht Fichte mit seiner Forderung nach Erschöpfung des aufzustellenden ersten Grundsatzes in der darauf aufbauenden Darstellung des Systems des menschlichen Wissens über das Vollständigkeitsideal der Ordnungswissenschaften des 18. Jahrhunderts hinaus. Das geschah dadurch, daß für die Vollständigkeit der abzuleitenden Sätze kein bloß formales, sondern ein gleichermaßen inhaltsbezogenes Kriterium angegeben wurde: Alle möglichen Sätze sind aus dem ersten Grundsatz zu deduzieren, und alle Sätze sollen auf diesen zurück verweisen. ${ }^{13}$ Manifestiert sich in dieser Reflexivität der Beziehungen Erster Grundsatz - abgeleitete Sätze der methodisch gewendete Begriff des Naturzwecks Kants, ${ }^{14}$ so führte Fichte erneut eine entscheidende Änderung ein: Um die Explikation der wesentlichen Strukturen begrifflich vollziehen zu können, ist eine Beziehung im Gefüge der darzustellenden Totalität begründet herauszuheben - für die Darstellung des Systems des menschlichen Wissens der Erste Grundsatz - , ohne daß diese deswegen schon das System selbst repräsentiert. Insofern in diesem Ersten Grundsatz als einem Widerspruchsverhältnis die Bestimmungen des Systems keimhaft angelegt sind, da doch die folgenden Sätze "gleichsam schon in ihm enthalten lagen, « ${ }^{15}$ ist er vor allem qualitativ bestimmt, ging es Fichte um die Deduktion des Inhalts des Wissens, d.h. in seinen wesentlichen, kategorialen Formen. Auf methodologischer Ebene formulierte der Philosoph so die Forderung an eine theoretische Darstellung, daß diese nicht vordergründig durch die Form bestimmt sei, sondern durch den Inhalt mit der diesem eigenen Form. ${ }^{16}$ Gleichwohl Fichte die Form lediglich Mittel und nicht mehr Zweck der Entfaltung der wissenschaftlichen Theorie war, so wenig galt sie ihm als beliebig; denn Inhalt und Form einer wissenschaftlichen Theorie bedingen sich, haben ihre Funktion in bezug aufeinander. Aber nicht mehr die Systemhaftigkeit schlechthin verbürgt die Gewißheit des Wissens, sondern allein der Ausgang von einem ersten, absoluten Grundsatz. Durch die inhaltliche Bestimmung des Ersten Grundsatzes wird zugleich die logisch-formale Abfolge der deduzierten Sätze bestimmt und damit deren Stellung im System. ${ }^{17}$

Was man lediglich als Verschärfung der logisch-methodischen Kriterien an den Systemaufbau der wissenschaftlichen Theorie werten könnte, erweist sich unter dem Gesichtspunkt der Dialektikentwicklung als entscheidender Fortschritt. Durch die qualitative Bestimmung des Ersten Grundsatzes als dialek-

\footnotetext{
13. Vgl.: Ebenda, S. 130.

14. Vgl.: I. Kant: Kritik der Urteilskraft, B $286 \mathrm{ff}$.

15. J. G. Fichte: Ueber den Begriff [...], GA I/2, 123

16. Vgl.: Ebenda, S. 115.

17. Vgl.: Ebenda, S. 121 .
} 
tisch-widersprüchliches Ausgangsverhältnis und die reflexive Begründung der Beziehung Erster Grundsatz und abgeleitete Sätze sind auch Anfang und Resultat der Darstellung aufeinander verwiesen. ${ }^{18}$ Jedoch nicht im Sinne einer formallogischen Identität; die Deduktion realisiert sich durch eine Konkretisierung des Ausgangsverhältnisses, wodurch Fichte die Vernunft als eine Totalität von Bestimmungen darzustellen vermag, deren Momente darin eine Genese oder Entwicklung vollziehen. Das strukturierte Wesen des Erkenntnisobjektes ist trotz der ihm eigenen unendlichen Beziehungsvielfalt in einer endlichen Zahl von Erkenntnisschritten auf der bestimmten historisch konkreten Stufe der Erkenntnis theoretisch reproduzierbar; trotz der Relativität menschlicher Erkenntnis weist dieses Wesen in einem bestimmten Umfang zugleich absolute Momente auf. Fichte gab damit unter Aufnahme und Weiterentwicklung der bei Kant in der Ableitung der Kategorien hervortretenden Triplizitätsstruktur einen entscheidenden Anstoß innerhalb des klassischen deutschen Idealismus hin zur Ausbildung der Philosophie als Entwicklungstheorie.

Die Notwendigkeit von Wissenschaft ergab sich für Fichte aus dem Unterschied von Wesen und Erscheinung des unabhängig vom wissenschaftlichen Erkennen existierenden Objektes. Das Wesen ist jedoch nicht mehr verstanden als ein Besonderes, verborgen Existierendes wie in der Tradition der Substanzmetaphysik des 17. und 18. Jahrhunderts. Statt dessen faßte Fichte das Wesen als die dem Objekt der Wissenschaftslehre - dem System des menschlichen Wissens ${ }^{19}$ — eigene Gesetzlichkeit, die auf vielfältige und widersprüchliche Weise erscheint. Dabei ist es ein nicht zu unterschätzendes Verdienst Fichtes, in der methodologischen Erörterung darauf hingewiesen zu haben, daß die im menschlichen Geiste vorkommenden Handlungen "nicht nothwendig [...] wirklich der Folge nach in jener systematischen Form, eine nach der andern, in unserm Geiste vorkommen; $[\ldots] \ll .{ }^{20}$ Der logische Ausgangspunkt für die theoretische Systementfaltung ist aber deswegen kein beliebiger im utilitaristischen Sinne, der keiner Begründung bedarf, und das als Erstes Erscheinende keineswegs auch das Erste, der Ausgangspunkt der systematischen Darstellung. Bekanntermaßen weitete Hegel diesen Gesichtspunkt in der Phänomenologie des Geistes aus, in der er in der Darstellung der histo-

18. Vgl.: Ebenda, S. 133.

19. Zum Problem des Verständnisses des letzten Grundes des Wissens und seiner durch Fichte in den späteren Darstellungen der Wissenschaftslehre vom Wissen her versuchten verstehenden Durchdringung vgl.: K. Hammacher: »Der Begriff des Wissens bei Fichte«, in: Zeitschrift für philosophische Forschung, Bd. 22. (1968).,H. 3, S. 345 ff.

20. Vgl. J.G. Fichte: Ueber den Begriff [...], GA I/2, 140. 
rischen Erscheinungsweisen des Geistes hinführt zum Ausgangsverhältnis für die Darstellung der Wissenschaft der Logik.

Die sich hiermit manifestierende latente Historisierung in der philosophischen Theorienbildung kommt in der Fichteschen Bestimmung der zwei Reihen noch expliziter zum Ausdruck: "Das Dargestellte und die Darstellung sind in zwei verschiedenen Reihen. ${ }^{21}$ Das Objekt und dessen theoretisch-begriffliche Repräsentation sind klar voneinander zu unterscheiden. Auf diese Weise vermochte Fichte den in der Begriffsschrift die Objektivität wissenschaftlichen Arbeitens akzentuierenden Gedanken, „Wir sind nicht Gesetzgeber des menschlichen Geistes, sondern seine Historiographen; [... « ${ }^{22}$ dahingehend auszuweiten, daß die Reihe der logisch-systematischen Darstellung der Handlungen des menschlichen Geistes seine »systematische Geschichte [...] in seinen allgemeinen Handlungsweisen $\ll^{23}$ und so zugleich »ein getroffenes Schema des menschl[ichen]. Geistes überhaupt « darstellt. ${ }^{24}$

Indem Fichte - so die hier vertretene These - seine methodologischen Überlegungen als Gesamtheit methodischer Konsequenzen des transzendentalphilosophischen Ansatzes entwickelte, war nun die Begründung der Möglichkeit von Wissenschaft nicht mehr ein vorwissenschaftlicher Akt, sondern selbst Moment des wissenschaftlichen Tuns und obliegt der »Wissenschaft von der Wissenschaft überhaupt« oder der Philosophie.

Auch wenn die methodologischen Erörterungen noch nicht die Philosophie selbst ausmachen, tragen sie nichtsdestoweniger philosophischen Charakter: "Eine Wissenschaft und die Kritik derselben, unterstützen und erklären sich wechselseitig $\ll{ }^{25}$ Die wissenschaftliche Methode ist nicht als ein Formalismus der Theorie des Objektes unabhängig von diesem vorausgesetzt gegeben, sondern aus der Theorie ableitbar, weil inhaltsbestimmt und geht wiederum als Moment in den Aufbau der Theorie ein. Mehr noch: Die Methode geht in ihr Gegenteil, die Theorie, über, wird über sie als Methode reflektiert.

21. Ebenda, S. 149.

22. Ebenda, S. 147.

23. J.G. Fichte: Von den Pflichten der Gelehrten. Vorlesungen und Entwürfe. [1794/95], GA II $/ 3,334$.

24. Ebenda, S. 328.

25. J. G. Fichte: Ueber den Begriff [...], GA I/2, S. 160 


\section{Philosophische Methodologie und Einzelwissenschaft}

Die neu gewonnene Auffassung vom organischen System oder der Totalität wird auf den Begriff der Wissenschaft und des Verhältnisses von Philosophie und Einzelwissenschaften bei gleichzeitiger Auflösung der hierarchischen Struktur des Systems der Wissenschaften angewandt: Die Metatheorie unterliegt folglich auch keinen anderen Gesetzen der Erkenntnis als die von ihr begründete bzw. kritisch reflektierte Theorie. ${ }^{26}$

Da die Wissenschaftslehre allen Wissenschaften die Form gibt, können diese nur in derselben Weise aus den Grundsätzen folgern wie jene. Somit erhebt sich die Frage, wie sich der Übergang zu den Einzelwissenschaften vollzieht und wodurch sich die Sätze der einen von den Sätzen der anderen unterscheiden. Das geschieht nach Auffassung Fichtes dadurch, daß der Urteilskraft die Aufgabe zukommt, »einen bestimmten Gegenstand nach einem bestimmten Gesetze zu beobachten«. Aufgabe der Naturwissenschaft sei es dagegen,

»jeden in der Erfahrung gegebnen Gegenstand an jedes in unserm Geiste gegebnes Naturgesetz zu halten, ist Grundsatz der Naturwissenschaft: sie besteht durchgängig aus Experimenten, (nicht aber aus dem leidenden Verhalten gegen die regellosen Einwirkungen der Natur auf uns) die man sich willkürlich aufgiebt, und denen die Natur entsprechen kann oder nicht $\left[\ldots .{ }^{27}{ }^{27}\right.$

Sieht man von der sowohl dem historischen Ausbildungsstand der Wissenschaften aber auch von der Fichtes Verständnis einer exponierten Stellung der Philosophie im System der Wissenschaften geschuldeten Verkennung des theoretischen Charakters der Naturwissenschaften ab, so ist an der gegebenen Bestimmung die Betonung des aktiven Charakters der wissenschaftlichen Arbeit, insbesondere durch das Experiment, hervorzuheben. Das Gesetz ist nicht in empiristischer Natur erfaßbar. Es bedarf dazu - modern gesprochen - der begrifflich-klassifikatorischen Repräsentation eines Problems als eines aktiven, kognitiven Vorgangs auf Seiten des Subjektes.

Auf der philosophischen Ebene kommt die Wissenschaftslehre ohne die Beziehung zu den Naturwissenschaften aus, sind die Denkbestimmungen doch a priori gegeben, werden sie in ihrer philosophischen Bestimmung herausgearbeitet. Und da sie a priori gegeben sind, gehen sie auch in dieser Weise in den Erklärungsprozeß der Naturwissenschaften ein, womit Fichte nach Auffassung Hegels die Sinnlichkeit intellektualisierte. Der Unterschied zwischen 
Einzelwissenschaft und Philosophie kann nicht grundsätzlicher Natur sein, da beide Momente eines einheitlichen Wissens sind, sie gleichen allgemeinen Gesetzen in der Erkenntnis genügen. Philosophie und Einzelwissenschaft unterscheiden sich dadurch, daß sie verschiedene Seiten des einen Objektes zum Gegenstand haben. Während die Philosophie nur das Notwendige enthalte (also die allgemeinen Gesetze und kategorialen Formen des Denkens, die als System erschöpfbar darstellbar sind), gingen alle übrigen Wissenschaften auf die Freiheit sowohl des Geistes als auch des Nicht-Ich aus. ${ }^{28}$ Die Bestimmung der freien Handlungen der empirischen Wissenschaften könne nicht in der Wissenschaftslehre, wohl aber in den einzelnen Wissenschaften geschehen.

»Demnach müßte im Grundsatze einer besondern Wissenschaft eine Handlung, die die Wissenschaftslehre frei gelassen hätte, bestimmt werden: Die Wissenschaftslehre gäbe dem Grundsatze das Nothwendige und die Freiheit überhaupt; die besondre Wissenschaft aber gäbe der Freiheit ihre Bestimmung; [...].« ${ }^{29}$

Der jeder speziellen Wissenschaft zugrunde liegende Satz ist also selbst nicht unbedingt, wird nicht durch diese Wissenschaft begründet, sondern durch die Wissenschaftslehre. Die einzelne Wissenschaft nimmt das Objekt, das NichtIch wie auch die Gesetze, mittels derer sie jenes beobachtet, notwendig als gegeben an. Dagegen hat die Wissenschaftslehre in ihrer Funktion als Methodologie die Berechtigung zu dieser Annahme aufzuklären, worin der Gedanke angelegt ist, daß Philosophie sich nicht im Gegensatz zur Erfahrung befindet. Während also die Einzelwissenschaften ihre Objekte ohne vorheriges Überdenken der Beziehungen zwischen Objekt und Subjekt im Erkenntnisprozeß untersuchen, sei gerade dieses Verhältnis der wichtigste Gegenstand der Philosophie. ${ }^{30}$ Denn alle Wissenschaft geht von der Voraussetzung der Erkennbarkeit ihrer Objekte aus. Aber die Wissenschaftslehre hat die Aufgabe, die Bedingungen für diese Voraussetzung mit all ihren Implikationen zu untersuchen. Dadurch erst wird die Vorstellung zu einem wissenschaftlichen Wissen. Indem die Wissenschaftslehre über die erkenntnistheoretischen Voraussetzungen einer jeden Wissenschaft reflektiert, wird sie eben zur »Wissenschaft der Wissenschaft $\ll$.

Die Fichtesche Idee, die Philosophie als allgemeine Methodologie der Wissenschaft zu entwickeln, intendiert zwar eine in sich unterschiedene Einheit

28. Vgl. Ebenda, S. 136, 142.

29. Vgl.: Ebenda, S. 134.

30. Vgl.: J.G. Fichte: Vergleichung des vom Hrn. Prof. Schmid aufgestellten Systems mit der Wissenschaftslehre, GA I/3, 248. 
der Wissenschaften, zugleich erhielt die Philosophie gegenüber den letzteren eine exponierte Stellung. Sie ist nicht durch die anderen Momente der Totalität der Wissenschaften, sondern a priori bestimmt und begründet daher die einzelnen Wissenschaften. Diesen Unterschied zwischen Philosophie und Einzelwissenschaft betonte Fichte dann auch sehr scharf:

"Die Wissenschaftslehre endet mit Aufstellung der reinen Empirie; sie bringt an's Licht, was wir wirklich erfahren können, nothwendig erfahren müssen, begründet sonach wahrhaftig die Möglichkeit aller Erfahrung. Ueber diese reine Erfahrung nun kann weiterhin räsonnirt, dieselbe combinirt, und systematisirt werden; und dies heißt mir Wissenschaft, welche da angeht, wo die Philosophie sich endet, und von unendlichem Umfange ist. Wissenschaft, und Philosophie sind mir sonach gar nicht einerlei, $[\ldots]$ sondern zweierlei; $[\ldots] . \star^{31}$

Pendant dieser Trennung ist andererseits Fichtes subjektiver Anspruch, die antithetische Methode nicht nur als spezifisch philosophische aufzufassen, sondern sie als universelle Methode zur Erzielung neuer Erkenntnisse auszuweisen. Daraus ergibt sich eben ihr methodologischer Charakter, die Möglichkeit und Notwendigkeit, einzelwissenschaftliches Vorgehen und dessen Resultate einer philosophischen Kritik zu unterwerfen. ${ }^{32}$ Die weiter wirkende Leistung dieser Wissenschaftsbegründung bestand nach meiner Auffassung darin, daß Fichte durch den Nachweis der Identität und des Unterschiedes in bezug auf die Methode und die Gesetze in den verschiedenen Wissenschaftsdisziplinen bei Betonung der besonderen Rolle der Philosophie unwissenschaftliche Konzepte wie beispielsweise den Psychologismus oder den "gedan[ken]losesten Empirismus « und den »Mysticism « ${ }^{33}$ aus der wissenschaftstheoretischen Diskussion verbannen wollte. Indem Fichte die Eigenständigkeit der Einzel-

31. Ebenda, S. 264. So war es nur konsequent, wenn Fichte an anderer Stelle schrieb: „Erfahrungen werden angeführt [...] zur Erläuterung keineswegs aber zum Beweise.« (J.G. Fichte: »Nachgelassene Schriften zu Platners Philosophischen Aphorismen« [1794-1812], GA II/4. S. 72.

32. Vgl.: J.G. Fichte: »Deduzierter Plan einer zu Berlin zu errichtenden höhern Lehranstalt, die in gehöriger Verbindung mit einer Akademie der Wissenschaften stehe «, in: Gelegentliche Gedanken über Universitäten, hrsg. v. E. Müller, Leipzig 1990, §§16 ff. (§16: „Sollte es nun doch zu einer solchen Kunst in der besondern Wissenschaft kommen (d.h. daß man versteht, was und wie man es macht - J.S.), so müßte dieselbe, unabhängig von ihrer Ausübung und noch ehe sie getrieben würde, verstanden, d.i. die Art und Weise der geistigen Tätigkeit, deren es dazu bedarf, erkannt werden, und so der allgemeine Begriff ihrer Kunst der Ausübung dieser Kunst selbst vorhergehen können. Nun ist dasjenige, was die gesamte geistige Tätigkeit, mithin auch alle besonderen und weiter bestimmten Äußerungen derselben wissenschaftlich erfaßt, die Philosophie:von philosophischer Kunstbildung aus müßte sonach den besonderen Wissenschaften ihre Kunst gegeben $[\ldots] . \ll$

33. J.G. Fichte: Brief Nr. 515 vom 23. 12. 1799, GA III/4, 171 
wissenschaften gegenüber der Philosophie unter der Voraussetzung der Einheit und Objektivität des Wissens begründete, vollzog er deren außerordentliche Aufwertung in der Wissenschaftsdiskussion. Denn noch bis in das 19. Jahrhundert war die Philosophische Fakultät ähnlich der mittelalterlichen Artistenfakultät oft kaum mehr als bloße Vorschule für die »oberen« Fakultäten. Erst im Verlaufe des 19. Jahrhunderts wurde die Philosophische Fakultät nicht nur zur gleichberechtigten, sondern zur führenden Fakultät, von der die wesentlichen Neuerungen im Wissenschaftsbetrieb nicht nur an der Jenaer Salana ausgingen. ${ }^{34}$

Fichte nahm diesen Prozeß gleichsam geistig vorweg, begründete sowohl der Philosophie als auch den nichtphilosophischen Wissenschaften einen jeweils eigenen Gegenstand und nahm im Gegenzug die »oberen« Fakultäten auf die Ebene der in der Philosophischen Fakultät etablierten Einzelwissenschaften zurück. Anders gesagt: Fichte gab in der wissenschaftstheoretischen Diskussion allen Wissenschaften den ihnen gebührenden Entwicklungsraum, der ihnen im Praktischen, in den überholten, an den Bedürfnissen der feudalen Kleinstaaten orientierten Universitätsstrukturen ermangelte.

Das Verhältnis von Philosophie zu den Einzelwissenschaften im System des menschlichen Wissens erfuhr bei Fichte eine Behandlung, die weit über das Beiläufige hinausging, indem es einen systembegründenden, konstitutiven Charakter erhielt. Diese Wertung läßt sich nicht nur für den Zeitraum der Ausbildung des Fichteschen transzendentalphilosophischen Ansatzes 1793/ 1794 treffen; mit der Entwicklung und Wandlung seines Systems rückte die methodologische Problematik immer wieder in das Blickfeld des Philosophen. Während aber die theoretische Entfaltung der transzendentalen Philosophie in einer Vielzahl von Schriften ihre Ausgestaltung, Umbildung und sogar Neufassung erfuhr, blieb die kleine Arbeit Ueber den Begriff der Wissenschaftslehre in den wesentlichen methodologischen Aussagen für Fichte vorausgesetzt. Die späteren wissenschaftstheoretischen Arbeiten revidierten nicht die

34. Bereits in den letzten zwanzig Jahren des 18. Jahrhunderts war nicht zuletzt durch den Einfluß Goethes der Versuch unternommen worden, der Jenaer Akademie neues Leben durch Neuberufungen sowie Neugründungen von wissenschaftlichen Anstalten - etwa des Botanischen Gartens, der Sozietät für Mineralogie oder der Errichtung eines chemischen Laboratoriums neues Leben zu verleihen. Die zunehmende Spezialisierung des wissenschaftlichen Betriebes in den Folgejahren des 19. Jahrhunderts fand ihren Ausdruck in der Erweiterung ordentlichèr Lehrstellen, vor allem innerhalb der Philosophischen Fakultät. 1821 besaß diese neun Ordinarien (theoretische Philosophie; praktische Philosophie; Beredsamkeit, Dichtkunst und Altertumskunde; Geschichte; Mathematik und Physik; Chemie; Staats- und Kameralwissenschaften; Mineralogie und Geologie), bis 1883 hatte sich die Zahl auf sechzehn erhöht. (Vgl.: M. Steinmetz: Geschichte der Universität Jena, Jena 1958, Bd. I, S. 316 f., 391 f.) 
formulierten methodologischen Positionen der Begriffsschrift; statt dessen gelangten in ihnen zusätzlich praktische Aspekte der philosophischen Lehrtätigkeit, der Vermittlung im pädagogischen und didaktischen Sinne sowie der Wissenschaftsorganisation in das Blickfeld.

Was Fichtes methodologische Erörterungen in der Bégriffsschrift auszeichnet, ist vor allem deren kognitiv-heuristischer Anspruch, den er mit den philosophisch-methodologischen Überlegungen für die einzelnen Wissenschaftsdisziplinen verknüpfte. Wenn Fichte später anregte, den erreichten Ausbildungsstand der Wissenschaften zu analysieren, mit Kritik zu begleiten, ${ }^{35}$ im Einzelfall gar den Mangel beklagte, daß es keine Philosophie der Mathematik gäbe, und er die daraus erwachsenden Nachteile für diese hervorhob, ${ }^{36}$ so nicht, um den Einzelwissenschaften konkrete Ergebnisse von philosophischer Seite vorzuschreiben, sondern um durch die Reflexion auf philosophischer Ebene die theoretisch-systematische Durchdringung der einzelnen Disziplin und ihres Gegenstandes zu befördern und Impulse für die Weiterentwicklung der Wissenschaften zu vermitteln.

Mit der Eingrenzung des Gegenstandes der Philosophie auf »das System des menschlichen Wissens überhaupt ${ }^{37}$ und der unternommenen Klassifizierung der Wissenschaftsdisziplinen suchte Fichte nicht nur nach einer Lösung formaler Problemstellungen der Systemkonstruktion. Ausgehend vom entwerfenden, tätigen Charakter des Subjektes und folglich auch der Wissenschaft ging es ihm dabei wesentlich um die Bestimmung der Rolle der Wissenschaft im gesellschaftlichen Leben, um die Entfaltung ihrer Potenzen für den Fortschritt der Menschheit. ${ }^{38}$ Insofern schließt die Ausarbeitung der Vorlesungen Über die Bestimmung des Gelehrten nicht nur zeitlich unmittelbar an die Grundlagen der gesammten Wissenschaftslehre und die Begriffsschrift an, sondern es besteht ein innerer logischer Zusammenhang, daß damit erneut über die soziale Funktion der Wissenschaft durch Fichte reflektiert wurde.

Während aber gerade in den Vorlesungen Über die Bestimmung des Gelehrten ein wesentliches Thema der Philosophie der Aufklärung, nämlich das der Allgemeingeltung als Problem der Vermittlung der Philosophie bis hin zur

35. Vgl.: J.G. Fichte: Jahrbücher der Kunst und Wissenschaft [1800], GA I/6, 425 f.; dazu: J. Stahl: "Ästhetik und Kunst in der Transzendentalphilosophie Johann Gottlieb Fichtes«, in: Philosophie und Kunst. Kultur und Ästhetik im Denken der deutschen Klassik. Collegium philosophicum Jenense, H. 7, hrsg. v. E. Lange, Weimar 1987, S. 83.

36. Vgl.: J.G. Fichte: Brief Nr. 515 vom 23.12.1799, GA III/4, 171.

37. J.G. Fichte: Ueber den Begriff [...], GA I/2, 129.

38. Vgl. ebenda, S. 153/154, wo Fichte bereits den grundlegenden Ansatz formuliert, den er dann in Die Bestimmung des Gelehrten weiter entwickelte. 
pädagogisch-didaktischen Dimension in geradezu pathetischer Weise aufgenommen wurde, vollzog Fichte in der Begriffsschrift hinsichtlich der Begründung der Allgemeingültigkeit des Wissens einen Paradigmawechsel gegenüber der aufklärerischen Diskussionslinie. Die noch bei Reinhold wesentlich formale Bestimmtheit des Systems des Wissens wurde durch Fichte aufgegeben zugunsten seiner inhaltlichen Beschaffenheit. ${ }^{39}$ Wird das Wesen von Wissenschaft aber in die Beschaffenheit des Inhalts und dessen Verhältnis zum erkennenden Bewußtsein gesetzt, so betrifft dies die Beziehung von Subjekt und Objekt. Gegenstand philosophischer Reflexionen sind somit auch nicht die formallogischen Strukturen; die formale Logik wird - wie bereits bei Kant und Maimon - in das Feld der Einzelwissenschaften verwiesen. Statt einer wesentlich formallogischen Begründung philosophischer Aussagen und dem vorrangigen Bezug auf die Mathematik, Physik und formale Logik tritt die Begründung der Möglichkeit der Konstruktion des Wissens. J. Manninen arbeitete die Erweiterung des Konstruktionsbegriffes durch Fichte über die Mathematik hinaus, die Aufhebung seiner bei Kant bezeichneten Beschränkung auf die Sinneswahrnehmung in eine universelle Fähigkeit des menschlichen Verstandes zur Begriffsbildung als eine der entscheidenden Leistungen der Fichteschen Philosophie heraus. ${ }^{40}$ Gerade im Konstruktionsbegriff manifestiert sich der entwerfende, tätige Charakter des Subjektes im Herausarbeiten der theoretischen-begrifflichen Fassung des Wesens eines Gegenstandes.

Mit der Rücknahme des Bezugs auf die Mathematik gewinnt in der Entfaltung des philosophischen Systems Fichtes die in der deutschen Philosophie insbesondere von Herder ausgehende Tendenz weiter an Gewicht, kultur- und sozialgeschichtliche Problemstellungen in den Vordergrund der philosophischen Erörterungen zu stellen. Die methodologische Orientierung am sozialtheoretischen Denken sowie der Erforschung des Denkprozesses selbst folgte der Intention Fichtes, den Menschen und die Gesellschaft aus der universell begriffenen Naturgesetzlichkeit herauszulösen und demgegenüber die Eigengesetzlichkeit, den besonderen Charakter menschlicher Freiheit und des sozialen Geschehens in einer einheitlichen philosophischen Ableitung, das gesamte Sein unter sich begreifend, zu begründen. Was sich externalistisch als Konsequenz des sozialpolitischen Engagements Fichtes für den gesellschaftlichen Fortschritt darstellt, erhielt unter Aufnahme des Kantschen Gedankens vom Primat der praktischen Vernunft in der Systemkonstruktion der Begriffs-

39. Vgl.: Ebenda, S. 113.

40. Vgl.: J. Manninen: „Relativität und Totalität des Wissens. Die Auseinandersetzung mit der Philosophie Hegels in Schweden«, in: Annalen der internationalen Gesellschaft für dialektische Philosophie Societas Hegeliana, Bd. III, Köln 1986, S. 220. 
schrift ${ }^{41}$ und in der späteren systematischen Ausführung seine folgerichtige Ausgestaltung.

In dieser Arbeit findet nicht die Ausarbeitung einer zeitlosen Methodologie statt, weil sie apriorische Momente menschlicher Erkenntnis bevorzugt herausarbeitet und den tätigen Charakter des erkennenden und handelnden Subjektes auf neue Weise begründet. Die durch Fichte darin erarbeiteten methodologischen Positionen geben einer Etappe der Wissenschaftsentwicklung Ausdruck, in der sich eine Vielzahl der grundlegenden Wissenschaftsdisziplinen konstituierten, indem sie den Übergang zu Theoriebildungsprozessen vollzogen, sich von anderen Disziplinen, in deren Schoße sie entstanden waren, sowohl nach der Seite des Gegenstandes als auch nach der Seite der zur Anwendung gelangenden besonderen Methoden emanzipierten. Zugleich ist diese Etappe der Wissenschaftsentwicklung dadurch charakterisierbar, daß sich Wissenschaft zunehmend als Element der bürgerlichen Kultur bei gleichzeitigem Abnabeln von den Bedürfnissen und Zwängen feudaler Kleinstaaterei in Deutschland entfaltete.

Fichte gab mit seinen in der Begriffsschrift formulierten methodologischen Positionen Anregungen, die weit über das unmittelbare Feld der Begründung seiner Transzendentalphilosophie hinaus wiesen - auch und besonders durch die spezifische, von Hegel als »schief « kritisierte Sicht auf den tätigen Charakter des Subjekts. Die — soweit ich sehe — sehr moderne Funktionsbestimmung der Philosophie gegenüber den nichtphilosophischen Wissenschaftsdisziplinen ist hier ebenso zu nennen wie Fichtes Beitrag zur Begründung der Dialektik als originärer philosophischer Methode, das im transzendentalen Ansatz akzentuierte Problem der Theorienbeladenheit wissenschaftlichen Erkennens und der Intersubjektivität der dabei zur Anwendung gelangenden Methoden oder das Herausheben der sozialen Verantwortung des Wissenschaftlers als »Lehrer des Menschengeschlechts« im zeitgeschichtlichen Prozeß. 